\title{
Incertidumbre de la Medición de Masa en la Determinación de los Parámetros de Consumo de Electrodos de Recargue
}

\author{
(Measurement Uncertainty of Mass in the Determination of the Consumption Parameters of Hardfacing Electrodes)
}

\author{
Rosenda Valdés Arencibial ${ }^{1}$ Antônio Piratelli-Filho², Amado Cruz-Crespo ${ }^{3}$,Eduardo Díaz Cedré ${ }^{4}$ \\ ${ }^{1}$ Universidade Federal de Uberlândia, Campus Santa Mônica. FEMEC. Bloco 1M, CEP 38400-902. \\ Uberlândia,Brasil.arvaldes@mecanica.ufu.br \\ ${ }^{2}$ Universidade de Brasília, Faculdade de Tecnologia, Depto. Engenharia Mecânica, 70910-900, Brasília, DF. Brasil.pirateli@unb.br \\ 3,4Universidad Central "Marta Abreu”de Las Villas, Cuba, acruz@uclv.edu.cu; cedre@.uclv.edu.cu
}

\begin{abstract}
Resumen
Este trabajo presenta la evaluación de la incertidumbre de las mediciones realizadas para la determinación de los parámetros de consumo de electrodos de recargue, dando énfasis a la medición de masa. Para ello fueron realizados tres depósitos a tres niveles de corriente (120 A, 145 A y 160 A, respectivamente), registrándose el tiempo de soldadura, la longitud del cordón, así como la masa inicial y final de las probetas y de los electrodos. A partir de los datos anteriores fueron determinados los parámetros de consumo. Fue calculada la incertidumbre asociada a la masa de las probetas, a la masa consumida del electrodo, a la masa depositada y a los parámetros de consumo. Al finalizar el trabajo se comprobó que el rendimiento del depósito muestra un comportamiento decreciente con la corriente, siendo el mejor resultado de $(74,12) \%$ para una corriente de soldadura de $120 \mathrm{~A}$. Los valores de incertidumbre expandida para el rendimiento varian entre $1,47 \%$ y 2,41\%, para la tasa de consumo fueron obtenidos valores entre $0,4 \mathrm{~g} / \mathrm{min}$ y $0,6 \mathrm{~g} /$ min, mientras que para la tasa de deposición la incertidumbre varía de 0,6 g/min a 0,7 g/min.
\end{abstract}

Palabras-clave: recargue por soldadura; trazabilidad; incertidumbre; medición de masa.

\begin{abstract}
This work presents the evaluation of the uncertainty of the measurements to determine the consumption parameters of hardfacing electrode, emphasizing the mass measurement. The experimentation was carried out obtaining deposits at three levels of current (120 A, $145 \mathrm{Ay} 160 \mathrm{~A}$ ) and measuring the time spent in welding, the length of the welds the test plate and electrode mass (initial and final). Based on these results, the consumption parameters were also determined. The uncertainty related to the measurements the mass of the samples, the mass consumption of the electrode, the deposited mass and consumption parameters was determined. The results showed that the deposition efficiency increase as a function of the current, turning the best result (74.12\%) at $120 \mathrm{~A}$. The expanded measurement uncertainty associated to consumption parameters changed between $1.47 \%$ and $2.41 \%$ for the deposition efficiency, $0.4 \mathrm{~g} / \mathrm{min}$ and $0.6 \mathrm{~g} / \mathrm{min}$ for the consumption rate vary of $0.6 \mathrm{~g} / \mathrm{min}$ to $0.7 \mathrm{~g} / \mathrm{min}$ for the deposition rate.
\end{abstract}

Key words: hardfacing by welding; traceability; uncertainty; mass measurement.

\section{Introducción}

Como resultado del desarrollo permanente de la ciencia y la tecnología, los equipos industriales trabajan cada vez en condiciones más severas. Ello está muy relacionado a la búsqueda constante de mayor productividad y de disminución de los tiempos para reparaciones y mantenimiento.

En este contexto, los procesos de recargue, en particular el recargue por soldadura, ocupa un lugar destacado, pues posibilita que piezas desgastadas sean reparadas y devueltas

Recebido em 28/08/2012. Texto final em 18/02/2013. al servicio. Dichas piezas pueden tener, incluso, una vida útil más prolongada que las originales como resultado de recubrimientos más compatibles con las condiciones específicas de los mecanismos de desgaste. Lo anterior, unido a la búsqueda global de mayores efectos económicos, ha llevado también a la aplicación por soldadura de recubrimientos duros sobre sustratos de materiales más baratos.

Estas aplicaciones han estado relacionadas con investigaciones encaminadas al mejoramiento de las propiedades de los depósitos obtenidos por soldadura, abarcando desde el desarrollo de nuevos consumibles hasta el perfeccionamiento de los procedimientos de aplicación. Sin embargo, para que los resultados de esas investigaciones sean confiables es necesario que las mediciones que fundamentan las mismas sean trazables a los estándares nacionales e internacionales de la magnitud evaluada.

De acuerdo con [1] la trazabilidad es definida como la 
propiedad de un resultado de medición por la cual este puede ser relacionado a una referencia establecida mediante una cadena ininterrumpida y documentada de calibraciones, cada una de las cuales contribuye a la incertidumbre de medida. Este concepto reúne dos aspectos importantes. El primero radica en la utilización de sistemas de medición y equipos calibrados. El atendimiento a este requisito es indispensable para la obtención de resultados confiables [2] y cuando no es tomado en cuenta puede colocar en duda la fiabilidad de los mismos como es destacado por [3].

El segundo aspecto se refiere a la declaración de la incertidumbre en todos los niveles de la cadena de trazabilidad, incluso del resultado de la medición [2]. De forma general, la incertidumbre está directamente relacionada con la fiabilidad del proceso de medición, y es definida como un parámetro no negativo, que caracteriza la dispersión de los posibles valores atribuidos a un mensurando, con base en las informaciones utilizadas [1]. El cálculo de este parámetro y su presentación es esencial para adecuar los resultados de las mediciones a los estándares actualmente en vigor.

Para el cálculo de la incertidumbre existe un procedimiento recomendado por [4] y aceptado internacionalmente. Se destaca que este parámetro posee la misma unidad de medida del mensurando, la cual debe ser expresada de forma adecuada [5] y en concordancia con el Sistema Internacional de Unidades [6].

Las investigaciones en el área de soldadura exigen la medición de diversas magnitudes y para ello son utilizados diferentes instrumentos de medición, entre ellos: pies de rey, proyectores de perfil, balanzas, sofisticados sistemas de medición con procesamiento computacional de imágenes, entre otros. Cada uno de estos sistemas de medición posee sus particularidades y por lo tanto las fuentes de errores son diferentes, también, el cálculo de la incertidumbre [7-9]. Un caso particular de esto lo constituye la evaluación del rendimiento de materiales de aporte donde se efectúan mediciones de masa, conforme a lo presentado por [10-15].

Es por ello que, este trabajo tiene como objetivo evaluar la incertidumbre de las mediciones realizadas para la determinación de los parámetros de consumo de electrodos de recargue, dando énfasis, a la medición de masa.

\section{Metodología}

\subsection{Determinación de los Parámetros de Consumo}

Las características económicas de los electrodos son evaluadas según [16] y diferentes parámetros son utilizados para este fin, entre ellos: coeficiente de fusión, coeficiente de depósito, productividad del proceso, coeficiente de pérdidas, eficiencia de depósito respecto al núcleo del electrodo, eficiencia del depósito sin considerar las pérdidas por colillas y eficiencia total del electrodo. Esta norma destaca como principales parámetros de consumo las tasas de fusión y de deposición.

Para determinar los parámetros de consumo fueron efectuados cordones con un electrodo de recargue comercial de mediana aleación, de $(4,02 \pm 0,10) \mathrm{mm}$ de diámetro y $(350 \pm 1)$ $\mathrm{mm}$ de largo. Los depósitos fueron realizados en posición plana, sobre chapas de acero AISI 1020 de $200 \times 75 \times 10 \mathrm{~mm}$. El proceso de deposición fue conducido por un soldador calificado en el proceso SMAW, empleando una fuente MANSFELD G 500. El tiempo de arco fue medido con un cronómetro con resolución de 1 segundo y la longitud del cordón con una regla de acero con resolución de $0,5 \mathrm{~mm}$ y rango de medición de $300 \mathrm{~mm}$. Previamente, las chapas y los electrodos fueron identificados y su masa medida en una balanza con resolución de $0,1 \mathrm{~g}$ a través de la medición directa. Entre una lectura y otra se verificó el cero de la balanza. Luego de obtenidos los depósitos, la masa de las chapas y de los restos no consumidos de los electrodos fue nuevamente medida. Cuidados adicionales fueron seguidos con relación al manoseo de los electrodos y a la limpieza de las probetas durante la medición. Para determinar la repetitividad de la balanza fue efectuada una serie de 3 lecturas de cada probeta en cada condición evaluada. Posteriormente fueron determinados los parámetros de consumo y la incertidumbre asociada.

\subsection{Evaluación de la incertidumbre}

La incertidumbre asociada a la medición de masa fue evaluada siguiendo las recomendaciones de [4]. En este artículo se presenta de forma detallada la metodología propuesta para este fin. Los valores obtenidos permitieron el cálculo de la incertidumbre de los parámetros de consumo (tasa de consumo, tasa de deposición y de rendimiento). Para cada mensurando de interés fueron identificadas las variables de influencia y propuesto un modelo matemático para la posterior aplicación de la ley de propagación de incertidumbres.

\subsubsection{Incertidumbre referente a la medición de masa}

La medición de masa es imprescindible para evaluar el electrodo en cuanto a parámetros de consumo, conforme muestran los trabajos publicados por [10-15]. Estos autores midieron la masa de los electrodos y de las probetas antes y después de la soldadura para evaluar la eficiencia del depósito.

La obtención de datos confiables durante la medición de masa exige: utilizar balanzas calibradas cuya resolución sea adecuada para el nivel de exactitud requerido, efectuar por lo menos tres mediciones para detectar posibles errores groseros y posibilitar un análisis estadístico, evaluar la incertidumbre de medición, efectuar las mediciones para valores de temperatura y humedad adecuados, entre otros.

Son diversas las variables que pueden contribuir para la incertidumbre de la medición de masa. Según [17] son ellas: variabilidad de las lecturas (expresada a través de la desviación estándar experimental), resolución de la balanza, incertidumbre de la calibración de la balanza, efectos térmicos provocados por la variación de la temperatura del aire durante la medición, efectos provocados por campos electromagnéticos, estabilidad de la balanza, o sea, la capacidad de conservar sus parámetros metrológicos con el transcurso del tiempo y error de excentricidad de la balanza. La presión atmosférica, la humedad relativa del aire y el empuje del aire constituyen, también, fuentes de incertidumbre [18]. Además de estas, otras variables pueden ser consideradas, tales como: no linealidad y variación 
del cero de la balanza.

Ciertamente, no es necesario considerar todas estas variables para evaluar la incertidumbre de la medición de masa, en una determinada aplicación. De forma general, se mide la masa de una gran cantidad de mensurandos, en diversas áreas del conocimiento y en diferentes niveles de exactitud. Así, se debe efectuar un estudio caso a caso para definir cuales variables serán consideradas. Los efectos relacionados a la temperatura, a los campos electromagnéticos y el error de excentricidad pueden ser excluidos del cálculo de la incertidumbre en la mayoría de los casos [17]. El empuje del aire, también, es frecuentemente despreciado en muchas aplicaciones, porque la densidad de las probetas normalmente es mucho mayor que la densidad del aire. Este efecto es consecuencia directa del principio de Arquímedes.

Para evaluar la incertidumbre de la medición de masa, en este trabajo, fueron consideradas las siguientes variables: corrección asociada a la desviación estándar de los valores del mensurando indicados por la balanza $\left(\ddot{\mathrm{s}} \mathrm{s}\left(\mathrm{L}_{\mathrm{B}}\right)\right.$; corrección debida a la resolución de la balanza (incremento digital) $\left(\Delta R_{B}\right)$; corrección asociada a la incertidumbre estándar de la calibración

de la balanza $\left(\Delta \mathbf{C}_{\mathrm{B}}\right)$; corrección debida a la variación del cero de la balanza $\left(\ddot{\mathrm{AC}}_{\mathrm{B}}\right)$; corrección debida a la no linealidad de la balanza $\left(\Delta \mathbf{N}_{B}\right)$ y corrección debida a la excentricidad de la balanza $\left(\Delta \mathrm{E}_{\mathrm{B}}\right)$.

Así, el modelo matemático propuesto para evaluar la incertidumbre de la medición de masa es dado en la Ecuación 1. Donde $\mathrm{M}$ representa la masa medida.

$M=\Delta s\left(L_{B}\right)+\Delta R_{B}+\Delta I C_{B}+\Delta C_{B}+\Delta N L_{B}+\Delta E_{B}$

La incertidumbre estándar de la dispersión de los valores del mensurando puede ser obtenida a través de una evaluación del Tipo A, considerando una distribución normal y n-1 grados de libertad, Ecuación 2. Se espera que $n$ lecturas sean efectuadas para posteriormente determinar el valor medio de la masa.

$u\left(\Delta s\left(L_{B}\right)\right)=\frac{s\left(L_{B}\right)}{\sqrt{n}}$

Donde $\mathrm{n}$ representa el número de lecturas realizadas y $\mathrm{s}\left(\mathrm{L}_{\mathrm{B}}\right)$ es la desviación estándar.

Es importante destacar que la evaluación del Tipo A de la incertidumbre estándar puede ser efectuada solamente cuando se posee una serie de observaciones repetidas de un mensurando, pues es obtenida a partir de un análisis estadístico [4]. Generalmente se adopta la distribución normal debido al carácter aleatorio del proceso de medición.

Por su parte, la incertidumbre estándar de la resolución de la balanza es obtenida a través de una evaluación del Tipo B, considerando una distribución rectangular de probabilidades.

Como la balanza utilizada es digital se puede aplicar la

Ecuación 3. Obsérvese que la resolución $\left(\mathrm{R}_{\mathrm{B}}\right)$ es dividida por 2 , pues este es el mayor error esperado durante la lectura de la indicación de la balanza debido al redondeo, siendo de $\pm 0,05 \mathrm{~g}$.
$u\left(\Delta R_{B}\right)=\frac{\left(R_{B} / 2\right)}{\sqrt{3}}$

Una evaluación del Tipo B de la incertidumbre estándar es efectuada cuando no se dispone de una serie de observaciones repetidas del mensurando, en estos casos no es posible aplicar una análisis estadística y la incertidumbre es obtenida por otros medios, tales como consideraciones de manuales, especificaciones de fabricantes, certificados de calibración o a partir de experiencias anteriores [4]. En este trabajo los valores referentes a las variables $\left(\mathrm{IC}_{\mathrm{B}}, \mathrm{Z}_{\mathrm{B}}, \mathrm{NL}_{\mathrm{B}}\right.$ y $\left.\mathrm{E}_{\mathrm{B}}\right)$ son extraídos del certificado de calibración de la balanza lo que justifica efectuar una evaluación del Tipo B para la determinación de la incertidumbre estándar asociada.

En función de la cantidad de información disponible y de la forma como esta es presentada pueden ser adoptadas diferentes distribuciones de probabilidad. La distribución rectangular, por ejemplo, es frecuentemente adoptada cuando se dispone de poca información sobre la variable en estudio [4].

La incertidumbre estándar de la calibración de la balanza es determinada considerándose una evaluación del Tipo B, Ecuación 4. En este caso se adopta una distribución normal, siendo justificado por el Teorema del Limite Central [4]. En la Ecuación 4, $\mathrm{IC}_{\mathrm{B}}$ representa la incertidumbre declarada en el certificado de calibración de la balanza. Generalmente es dada como una incertidumbre expandida para un factor de cobertura (k) y un nivel de cobertura determinado.

$u\left(\Delta I C_{B}\right)=\frac{I C_{B}}{k}$

La incertidumbre estándar debida a la variación del cero de la balanza, también es efectuada considerándose una evaluación del Tipo B, Ecuación 5, donde $\mathrm{C}_{\mathrm{B}}$ es la variación del cero. La variación del cero representa el error observado cuando el valor medido especificado es cero [1].

$u\left(\Delta C_{B}\right)=\frac{C_{B}}{\sqrt{3}}$

La incertidumbre estándar debida a la no linealidad de la balanza es determinada por la Ecuación 6 , donde $\mathrm{C}_{\mathrm{B}}$ es el valor declarado de la no linealidad de la balanza. Este parámetro expresa la máxima desviación entre la curva de respuesta real de la balanza y una línea reta.

$u\left(\Delta N L_{B}\right)=\frac{N L_{B}}{\sqrt{3}}$

La incertidumbre estándar de la excentricidad de la balanza es dada por la Ecuación 7. Según [16] la excentricidad de una balanza es considerada como la mayor diferencia de errores de indicación entre las esquinas y el centro por aplicación excéntrica de una carga.

$u\left(\Delta E_{B}\right)=\frac{E_{B}}{\sqrt{3}}$ 
Aplicando la ley de propagación de incertidumbre en la Ecuación 1 se obtiene la Ecuación 8, que permite calcular la incertidumbre estándar combinada de la medición.

$$
\begin{aligned}
u_{c}^{2}(M)= & \left(\frac{\partial(M)}{\partial \Delta s\left(L_{B}\right)}\right)^{2} \cdot u^{2}\left(\Delta s\left(L_{B}\right)\right)+\left(\frac{\partial(M)}{\partial\left(\Delta R_{B}\right)}\right)^{2} \cdot u^{2}\left(\Delta R_{B}\right)+\left(\frac{\partial(M)}{\partial\left(\Delta I C_{B}\right)}\right)^{2} \cdot u^{2}\left(\Delta I C_{B}\right)+ \\
& +\left(\frac{\partial(M)}{\partial\left(\Delta C_{B}\right)}\right)^{2} \cdot u^{2}\left(\Delta C_{B}\right)+\left(\frac{\partial(M)}{\partial\left(\Delta N L_{B}\right)}\right)^{2} \cdot u^{2}\left(\Delta N L_{B}\right)+\left(\frac{\partial(M)}{\partial\left(\Delta E_{B}\right)}\right)^{2} \cdot u^{2}\left(\Delta E_{B}\right)
\end{aligned}
$$

En la Ecuación 8, las derivadas parciales representan los coeficientes de sensibilidad. Los mismos indican cual es la porción de contribución de cada variable de entrada en la incertidumbre final. Como el modelo matemático de la medición, Ecuación 1, es el resultado de la suma algébrica de las correcciones asociadas a las variables de influencia, entonces todas las derivadas parciales son unitarias. Son ellas:

$$
\left(\frac{\partial(M)}{\partial \Delta s\left(L_{B}\right)}\right)=\left(\frac{\partial(M)}{\partial\left(\Delta R_{B}\right)}\right)=\left(\frac{\partial(M)}{\partial\left(\Delta I C_{B}\right)}\right)=\left(\frac{\partial(M)}{\partial\left(\Delta C_{B}\right)}\right)=\left(\frac{\partial(M)}{\partial\left(\Delta N L_{B}\right)}\right)=\left(\frac{\partial(M)}{\partial\left(\Delta E_{B}\right)}\right)=1
$$

La incertidumbre expandida debe ser calculada utilizando la Ecuación 9 [4]. Donde $u_{c}$ es la incertidumbre estándar calculada por la Ecuación 8 y k es el factor de cobertura.

$$
U(M)=u_{c}(M) \cdot k
$$

El valor de $\mathrm{k}$ depende del número de grados de libertad efectivos de la medición y de la probabilidad de cobertura deseada y su valor puede ser obtenido de la Tabla de la distribución T-student. Por su parte, el grado de libertad efectivo $\left(v_{\mathrm{ef}}\right)$, es calculado por la Ecuación 10 [4].

$$
v_{e f}=\frac{u_{c}^{4}(y)}{\sum_{i=1}^{N} \frac{u^{4}\left(y_{i}\right)}{v_{i}}}=\frac{u_{c}^{4}(y)}{\sum_{i=1}^{N} \frac{\left(u\left(x_{i}\right) \cdot c_{i}\left(x_{i}\right)\right)^{4}}{v_{i}}}
$$

Donde: $N$ es el número de variables de entrada, $v_{i}$ es el número de grados de libertad de cada variable de entrada, $\mathrm{u}\left(\mathrm{y}_{\mathrm{i}}\right)$ es la incertidumbre estándar de cada variable de entrada en la unidad del mensurando o variable de salida, $\mathrm{u}\left(\mathrm{x}_{\mathrm{i}}\right)$ es la incertidumbre estándar de cada variable de entrada $\mathrm{y}_{\mathrm{i}}\left(\mathrm{x}_{\mathrm{i}}\right)$ representa el coeficiente de sensibilidad del mensurando en relación a cada variable de entrada $x_{i}$.

La masa consumida del electrodo, la masa del metal depositada, el rendimiento y las tasas de consumo y de deposición son calculados por las siguientes ecuaciones.

$$
\begin{aligned}
& m_{c}=M_{f e l}-M_{i e l} \\
& M_{d}=M_{f c h}-M_{i c h} \\
& R=\left(M_{d} / m_{c}\right) \cdot 100 \\
& T_{c}=\frac{m_{c}}{t_{s}}
\end{aligned}
$$

$T_{d}=\frac{M_{d}}{t_{s}}$

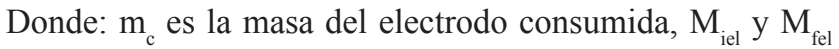
son los valores medios de masa inicial y final del electrodo, $\mathrm{M}_{\mathrm{d}}$ es la masa del depósito, $\mathrm{M}_{\text {ich }} \mathrm{y}_{\mathrm{M}_{\text {fch }}}$ son la masa inicial y final de

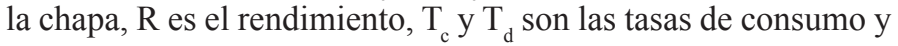
de deposición y $\mathrm{t}_{\mathrm{s}}$ es el tiempo de soldadura.

Aplicando la ley de propagación de incertidumbre en las Ecuaciones 11-15 y calculando las derivadas parciales se obtienen las Ecuaciones 16-20, que permiten calcular la incertidumbre estándar combinada asociada a estos parámetros. Se destaca que las variables de entrada en las Ecuaciones 11-13 son correlacionadas, pues fueron medidas utilizando el mismo sistema de medición y que la correlación es positiva y unitaria.

$$
\begin{aligned}
& u_{c}\left(m_{c}\right)=\sqrt{u^{2}\left(M_{f e l}\right)+u^{2}\left(M_{i e l}\right)} \\
& u_{c}\left(M_{d}\right)=\sqrt{u^{2}\left(M_{f c h}\right)+u^{2}\left(M_{i c h}\right)}
\end{aligned}
$$

$$
\begin{aligned}
& u_{c}(R)=\sqrt{\left(\frac{100}{m_{c}}\right)^{2} \cdot u^{2}\left(M_{d}\right)+\left(-\frac{100 M_{d}}{m_{c}{ }^{2}}\right)^{2} \cdot u^{2}\left(m_{c}\right)} \\
& u_{c}\left(T_{c}\right)=\sqrt{\left(\frac{1}{t_{s}}\right)^{2} \cdot u^{2}\left(m_{c}\right)+\left(-\frac{m_{c}}{t_{s}{ }^{2}}\right)^{2} \cdot u^{2}\left(t_{s}\right)}
\end{aligned}
$$

$$
u_{c}\left(T_{d}\right)=\sqrt{\left(\frac{1}{t_{s}}\right)^{2} \cdot u^{2}\left(M_{d}\right)+\left(-\frac{M_{d}}{t_{s}{ }^{2}}\right)^{2} \cdot u^{2}\left(t_{s}\right)}
$$

El modelo matemático utilizado para la determinación de la incertidumbre de la medición del tiempo del arco es presentado en la Ecuación 21. Mientras que la Ecuación 22 permite determinar la incertidumbre estándar combinada.

$$
\begin{aligned}
& t_{s}=\Delta s\left(t_{s}\right)+\Delta R_{\text {Cron }}+\Delta I C_{\text {Cron }} \\
& u_{c}\left(T_{s}\right)=\sqrt{\cdot u^{2}\left(\Delta s\left(T_{s}\right)+u^{2}\left(\Delta R_{\text {Cron }}\right)+u^{2}\left(\Delta C_{\text {Cron }}\right)\right.}
\end{aligned}
$$

Donde $\Delta s\left(t_{s}\right)$ es la corrección asociada a la desviación estándar de los valores del mensurando indicados por el cronometro, $\Delta R_{\text {Cron }}$ es la corrección debida a la resolución del cronometro y $\Delta C_{C r o n}$ es la corrección asociada a la incertidumbre estándar de la calibración del cronometro. Esta última fue retirada del certificado de calibración del cronómetro.

\section{Resultados y Discusión}

En la Tabla 1 se muestran los valores de masa obtenidos en cada medición para cada condición evaluada, acompañados de la media aritmética y la desviación estándar. 
Tabla 1. Resultados de la medición de masa

\begin{tabular}{c|c|c|c|c|c|c}
\hline $\mathrm{I}_{\mathrm{s}}(\mathrm{A})$ & & $\mathrm{L} 1(\mathrm{~g})$ & $\mathrm{L} 2(\mathrm{~g})$ & $\mathrm{L} 3(\mathrm{~g})$ & Media $(\mathrm{g})$ & $\mathrm{s}(\mathrm{g})$ \\
\hline \multirow{4}{*}{120} & $\mathrm{M}_{\text {iel }}$ & 70,9 & 71,1 & 71,3 & 71,1 & 0,20 \\
\cline { 2 - 7 } & $\mathrm{M}_{\text {fel }}$ & 34,2 & 33,9 & 34,0 & 34,0 & 0,15 \\
\cline { 2 - 7 } & $\mathrm{M}_{\text {ich }}$ & 325,9 & 326,0 & 326,1 & 326,0 & 0,10 \\
\cline { 2 - 7 } & $\mathrm{M}_{\text {fch }}$ & 353,7 & 353,5 & 353,3 & 353,5 & 0,20 \\
\hline \multirow{4}{*}{145} & $\mathrm{M}_{\text {iel }}$ & 71,7 & 71,4 & 71,4 & 71,5 & 0,17 \\
\cline { 2 - 7 } & $\mathrm{M}_{\text {fel }}$ & 38,8 & 39,0 & 39,2 & 39,0 & 0,20 \\
\cline { 2 - 7 } & $\mathrm{M}_{\text {ich }}$ & 316,9 & 317,1 & 317,3 & 317,1 & 0,20 \\
\cline { 2 - 7 } & $\mathrm{M}_{\text {fch }}$ & 340,4 & 340,1 & 340,1 & 340,2 & 0,17 \\
\hline \multirow{4}{*}{160} & $\mathrm{M}_{\text {iel }}$ & 70,9 & 71,1 & 71,0 & 71,0 & 0,10 \\
\cline { 2 - 7 } & $\mathrm{M}_{\text {fel }}$ & 49,0 & 49,1 & 49,2 & 49,1 & 0,10 \\
\cline { 2 - 7 } & $\mathrm{M}_{\text {ich }}$ & 344,3 & 344,0 & 344,3 & 344,2 & 0,17 \\
\cline { 2 - 6 } & $\mathrm{M}_{\text {fch }}$ & 357,8 & 358,0 & 358,2 & 358,0 & 0,20 \\
\hline
\end{tabular}

En la Tabla 1 fue añadido un dígito a los valores de la desviación estándar con la finalidad de facilitar la comparación de los mismos. Obsérvese que la desviación estándar varía entre 0,10 g y 0,20 g para todos los casos. Un aumento del tamaño de la muestra debe revelar valores de desviación estándar menores y más homogéneos.

En la Tabla 2 se muestran, para los diferentes valores de corriente de soldadura $I_{s}$, los valores medios de: masa inicial $M_{\text {iel }}$ y final $M_{\text {fel }}$ del electrodo, masa inicial $M_{\text {ich }}$ y final $M_{\text {fch }}$ de la chapa, tiempo de soldadura $\mathrm{t}_{\mathrm{s}} \mathrm{y}$ longitud del cordón $\mathrm{l}_{\mathrm{c}}$.

Tabla 2. Valores medios de masa, tiempo de soldadura y longitud del cordón

\begin{tabular}{c|c|c|c|c|c|c}
\hline $\begin{array}{c}\mathrm{I}_{\mathrm{s}} \\
(\mathrm{A})\end{array}$ & $\begin{array}{c}\mathrm{M}_{\text {iel }} \\
(\mathrm{g})\end{array}$ & $\begin{array}{c}\mathrm{M}_{\text {fel }} \\
(\mathrm{g})\end{array}$ & $\begin{array}{c}\mathrm{M}_{\text {ich }} \\
(\mathrm{g})\end{array}$ & $\begin{array}{c}\mathrm{M}_{\text {fch }} \\
(\mathrm{g})\end{array}$ & $\begin{array}{c}\mathrm{t}_{\mathrm{s}} \\
(\mathrm{min})\end{array}$ & $\begin{array}{c}\mathrm{c}_{\mathrm{c}} \\
(\mathrm{mm})\end{array}$ \\
\hline 120 & 71,1 & 34,0 & 326,0 & 353,5 & 1,50 & 132,0 \\
\hline 145 & 71,5 & 39,0 & 317,1 & 340,2 & 1,16 & 115,0 \\
\hline 160 & 71,0 & 49,1 & 344,2 & 358,0 & 0,73 & 90,0 \\
\hline
\end{tabular}

\subsection{Evaluación de la incertidumbre}

La incertidumbre asociada a la medición de masa fue calculada aplicando la metodología abordada en el epígrafe 2.2.1. La Tabla 3 muestra, de forma resumida, los resultados de incertidumbre obtenidos. Es conveniente presentar el cálculo de la incertidumbre de esta manera para facilitar el análisis de los resultados, conforme a lo recomendado por [4]. Obsérvese que la referida tabla muestra todos los datos relevantes para el cálculo de la incertidumbre y todos los resultados obtenidos, siendo de gran utilidad durante la identificación de las variables que más contribuyen para la incertidumbre final.

Tabla 3. Valores de incertidumbre para la medición de la masa inicial del electrodo para $120 \mathrm{~A}$

\begin{tabular}{|c|c|c|c|c|c|c|}
\hline Magnitud & Estimativa & TE & DP & GL & $\mathrm{CS}$ & $\mathrm{u}(\mathrm{g})$ \\
\hline$\Delta \mathrm{s}(\mathrm{L})$ & $0,20 \mathrm{~g}$ & $\mathrm{~A}$ & $\mathrm{~N}$ & 2 & 1 & 0,1155 \\
\hline$\Delta \mathrm{R}(\mathrm{b})$ & $0,1 \mathrm{~g}$ & B & $\mathrm{R}$ & $\infty$ & 1 & 0,0289 \\
\hline$\Delta \mathrm{C}(\mathrm{b})$ & 0 & B & $\mathrm{R}$ & $\infty$ & 1 & 0 \\
\hline$\Delta \mathrm{NL}(\mathrm{b})$ & $0,1 \mathrm{~g}$ & B & $\mathrm{R}$ & $\infty$ & 1 & 0,0577 \\
\hline$\Delta \mathrm{EX}(\mathrm{b})$ & $0,2 \mathrm{~g}$ & B & $\mathrm{R}$ & $\infty$ & 1 & 0,1155 \\
\hline$\Delta \mathrm{IC}(\mathrm{b})$ & $0,05 \mathrm{~g}$ & B & $\mathrm{N}$ & 100 & 1 & 0,0500 \\
\hline \multicolumn{6}{|c|}{ Incertidumbre estándar combinada $\left(\mathrm{u}_{\mathrm{c}}\right)$ en $\mathrm{g}$} & 0,1826 \\
\hline \multicolumn{6}{|c|}{ Grado de libertad efectivo $v_{\text {ef }}$} & 12,49 \\
\hline \multicolumn{6}{|c|}{ Factor de cobertura $\mathrm{k}$} & 2,18 \\
\hline \multicolumn{6}{|c|}{ Incertidumbre expandida $(\mathrm{U})$ en $\mathrm{g}$} & 0,3981 \\
\hline
\end{tabular}

En la Tabla 3: TE representa el Tipo de evaluación, DP es la distribución de probabilidad adoptada, GL es el número de grados de libertad, CS es el coeficiente de sensibilidad y u es la incertidumbre estándar. Obsérvese que fueron añadidos 3 dígitos a los valores de incertidumbre estándar y de incertidumbre estándar combinada con el objetivo de disminuir los errores debidos al redondeo.

Al finalizar los cálculos, el valor de la incertidumbre expandida debe ser redondeado respetando el número de dígitos significativos de la medición, los cuales dependen de la resolución de la balanza utilizada $(0,1 \mathrm{~g})$. El redondeo de los valores de incertidumbre deberá ser efectuado siempre por exceso, salvo en aquellos casos en que el crecimiento introducido sea superior a $95 \%$ [18].

Se puede concluir a partir de la Tabla 3 que la incertidumbre expandida asociada a la medición de la masa inicial del electrodo es de $0,4 \mathrm{~g}$ para $\mathrm{k}$ igual a 2,18 y $95 \%$ de cobertura. De esta forma se tiene que la masa inicial del electrodo es, en media, de $(71,1 \pm 0,4) \mathrm{g}$ para $120 \mathrm{~A}$.

La variable que más contribuyó, en este caso, para la incertidumbre final de la medición de la masa fue la desviación estándar de las lecturas seguida de la excentricidad de la balanza. La incertidumbre estándar asociada a la variabilidad de las lecturas puede ser disminuida aumentando el tamaño de la muestra (número de lecturas).

De forma similar fue calculada la incertidumbre para la medición de la masa final del electrodo, masa inicial de la chapa y masa final de la chapa para la corriente de 120 A. Las Tablas 4-7 muestran los resultados obtenidos. 
Tabla 4. Valores de incertidumbre para la medición de la masa final del electrodo para $120 \mathrm{~A}$

\begin{tabular}{|c|c|c|c|c|c|c|}
\hline Magnitud & Estimativa & $\mathrm{TE}$ & DP & GL & $\mathrm{CS}$ & $\mathrm{u}(\mathrm{g})$ \\
\hline$\Delta \mathrm{s}(\mathrm{L})$ & $0,15 \mathrm{~g}$ & $\mathrm{~A}$ & $\mathrm{~N}$ & 2 & 1 & 0,0882 \\
\hline$\Delta \mathrm{R}(\mathrm{b})$ & $0,1 \mathrm{~g}$ & B & $\mathrm{R}$ & $\infty$ & 1 & 0,0289 \\
\hline$\Delta \mathrm{C}(\mathrm{b})$ & 0 & B & $\mathrm{R}$ & $\infty$ & 1 & 0 \\
\hline$\Delta \mathrm{NL}(\mathrm{b})$ & $0,1 \mathrm{~g}$ & $\mathrm{~B}$ & $\mathrm{R}$ & $\infty$ & 1 & 0,0577 \\
\hline$\Delta \mathrm{EX}(\mathrm{b})$ & $0,1147 \mathrm{~g}$ & B & $\mathrm{R}$ & $\infty$ & 1 & 0,1155 \\
\hline$\Delta \mathrm{IC}(\mathrm{b})$ & $0,05 \mathrm{~g}$ & B & $\mathrm{N}$ & 100 & 1 & 0,0500 \\
\hline \multicolumn{6}{|c|}{ Incertidumbre estándar combinada $\left(u_{c}\right)$ en $g$} & 0,1667 \\
\hline \multicolumn{6}{|c|}{ Grado de libertad efectivo $v_{\text {ef }}$} & 25,46 \\
\hline \multicolumn{6}{|c|}{ Factor de cobertura $\mathrm{k}$} & 2,06 \\
\hline \multicolumn{6}{|c|}{ Incertidumbre expandida (U) en $g$} & 0,3434 \\
\hline
\end{tabular}

A partir de la Tabla 4 se tiene que la incertidumbre expandida asociada a la medición de la masa final del electrodo es de 0,4 g para k igual a 2,06 y $95 \%$ de cobertura, consecuentemente, el resultado de la medición de masa, en este caso, debe ser presentado como $(34,0 \pm 0,4) \mathrm{g}$.

Tabla 5. Valores de incertidumbre para la medición de la masa inicial de la chapa para $120 \mathrm{~A}$

\begin{tabular}{|c|c|c|c|c|c|c|}
\hline Magnitud & Estimativa & $\mathrm{TE}$ & DP & GL & $\mathrm{CS}$ & $\mathrm{u}(\mathrm{g})$ \\
\hline$\Delta \mathrm{s}(\mathrm{L})$ & $0,10 \mathrm{~g}$ & A & $\mathrm{N}$ & 2 & 1 & 0,0577 \\
\hline$\Delta \mathrm{R}(\mathrm{b})$ & $0,1 \mathrm{~g}$ & $\mathrm{~B}$ & $\mathrm{R}$ & $\infty$ & 1 & 0,0289 \\
\hline$\Delta \mathrm{C}(\mathrm{b})$ & 0 & $\mathrm{~B}$ & $\mathrm{R}$ & $\infty$ & 1 & 0 \\
\hline$\Delta \mathrm{NL}(\mathrm{b})$ & $0,1 \mathrm{~g}$ & B & $\mathrm{R}$ & $\infty$ & 1 & 0,0577 \\
\hline$\Delta \mathrm{EX}(\mathrm{b})$ & $0,1147 \mathrm{~g}$ & $\mathrm{~B}$ & $\mathrm{R}$ & $\infty$ & 1 & 0,1155 \\
\hline$\Delta \mathrm{IC}(\mathrm{b})$ & $0,05 \mathrm{~g}$ & $\mathrm{~B}$ & $\mathrm{~N}$ & 100 & 1 & 0,0500 \\
\hline \multicolumn{6}{|c|}{ Incertidumbre estándar combinada $\left(u_{c}\right)$ en $g$} & 0,1528 \\
\hline \multicolumn{6}{|c|}{ Grado de libertad efectivo $v_{\text {ef }}$} & 96,91 \\
\hline \multicolumn{6}{|c|}{ Factor de cobertura $\mathrm{k}$} & 2,00 \\
\hline \multicolumn{6}{|c|}{ Incertidumbre expandida (U) en $g$} & 0,3056 \\
\hline
\end{tabular}

La medición de la masa inicial de la chapa presenta una incertidumbre expandida de $0,3 \mathrm{~g}$ para $\mathrm{k}$ igual a 2,00 y $95 \%$ de cobertura, consecuentemente, el resultado de la medición de masa es de $(326,0 \pm 0,3) \mathrm{g}$.

La medición de la masa final de la chapa presenta una incertidumbre expandida de $0,4 \mathrm{~g}$ para $\mathrm{k}$ igual a 2,18 y $95 \%$ de cobertura, así, el resultado de la medición de la masa es de $(353,5 \pm 0,4) \mathrm{g}$.

A partir de las Tablas 3 a 6 se concluye que la incertidumbre expandida asociada a la medición de la masa, para $120 \mathrm{~A}$, asume valores entre $0,3 \mathrm{~g}$ y $0,4 \mathrm{~g}$ con factores de cobertura próximos de 2 . Las variables que más contribuyeron para la incertidumbre final de la medición fueron la desviación estándar de las lecturas y el error de excentricidad de la balanza. Resultados similares fueron obtenidos para los tres niveles de corriente considerados, como muestra la Figura 1. Para todos los casos el nivel de cobertura es de $95 \%$.

Tabla 6. Valores de incertidumbre para la medición de la masa final de la chapa para $120 \mathrm{~A}$

\begin{tabular}{|c|c|c|c|c|c|c|}
\hline Magnitud & Estimativa & $\mathrm{TE}$ & DP & GL & $\mathrm{CS}$ & $\mathrm{u}(\mathrm{g})$ \\
\hline$\Delta \mathrm{s}(\mathrm{L})$ & $0,20 \mathrm{~g}$ & A & $\mathrm{N}$ & 2 & 1 & 0,1155 \\
\hline$\Delta \mathrm{R}(\mathrm{b})$ & $0,1 \mathrm{~g}$ & $\mathrm{~B}$ & $\mathrm{R}$ & $\infty$ & 1 & 0,0289 \\
\hline$\Delta \mathrm{C}(\mathrm{b})$ & 0 & B & $\mathrm{R}$ & $\infty$ & 1 & 0 \\
\hline$\Delta \mathrm{NL}(\mathrm{b})$ & $0,1 \mathrm{~g}$ & B & $\mathrm{R}$ & $\infty$ & 1 & 0,0577 \\
\hline$\Delta \mathrm{EX}(\mathrm{b})$ & $0,1147 \mathrm{~g}$ & $\mathrm{~B}$ & $\mathrm{R}$ & $\infty$ & 1 & 0,1155 \\
\hline$\Delta \mathrm{IC}(\mathrm{b})$ & $0,05 \mathrm{~g}$ & $\mathrm{~B}$ & $\mathrm{~N}$ & 100 & 1 & 0,0500 \\
\hline \multicolumn{6}{|c|}{ Incertidumbre estándar combinada $\left(\mathrm{u}_{\mathrm{c}}\right)$ en $\mathrm{g}$} & 0,1826 \\
\hline \multicolumn{6}{|c|}{ Grado de libertad efectivo $v_{\text {ef }}$} & 12,49 \\
\hline \multicolumn{6}{|c|}{ Factor de cobertura $\mathrm{k}$} & 2,18 \\
\hline \multicolumn{6}{|c|}{ Incertidumbre expandida (U) en $g$} & 0,3980 \\
\hline
\end{tabular}

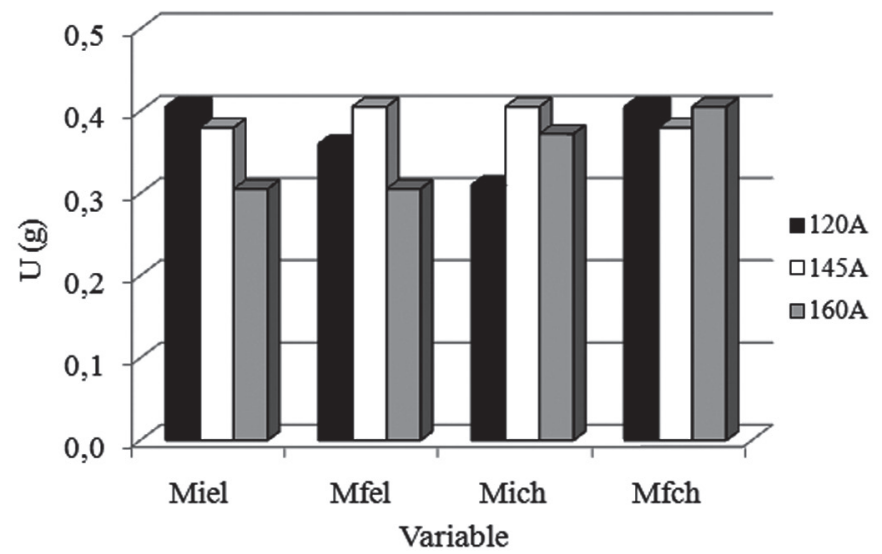

Figura 1. Incertidumbre expandida de la medición de masa $(95 \%)$

\subsection{Parámetros de consumo}

En base a los datos mostrados en la Tabla 2, fueron calculados la masa del electrodo consumida $\mathrm{m}_{\mathrm{c}}$, la masa del depósito $\mathrm{M}_{\mathrm{d}} \mathrm{y}$ los parámetros de consumo (rendimiento $\mathrm{R}$, tasa de consumo $\mathrm{T}_{\mathrm{c}}$ y tasa de deposición $\mathrm{T}_{\mathrm{d}}$ ). Los mismos son mostrados en la Tabla 7.

Tabla 7. Masa consumida, masa del depósito y parámetros de consumo

\begin{tabular}{c|c|c|c|c|c}
\hline $\begin{array}{c}\mathrm{I}_{\mathrm{s}} \\
(\mathrm{A})\end{array}$ & $\begin{array}{c}\mathrm{m}_{\mathrm{c}} \\
(\mathrm{g})\end{array}$ & $\begin{array}{c}\mathrm{M}_{\mathrm{d}} \\
(\mathrm{g})\end{array}$ & $\begin{array}{c}\mathrm{R} \\
(\%)\end{array}$ & $\begin{array}{c}\mathrm{T}_{\mathrm{c}} \\
(\mathrm{g} / \mathrm{min})\end{array}$ & $\begin{array}{c}\mathrm{T}_{\mathrm{d}}(\mathrm{g} / \\
\mathrm{min})\end{array}$ \\
\hline 120 & 37,1 & 27,5 & 74,12 & 24,7 & 18,3 \\
\hline 145 & 32,5 & 23,1 & 71,08 & 28,0 & 19,9 \\
\hline 160 & 21,9 & 13,8 & 63,01 & 30,0 & 18,9 \\
\hline
\end{tabular}


En la Tabla 7 se observa que el Rendimiento superior se obtiene con la menor intensidad de corriente de soldadura. Por tanto, desde este punto de vista el menor valor de corriente ofrece los mejores resultados de aplicación de recargue con el electrodo utilizado. Este hecho, que favorece el rendimiento para bajas corrientes $(120 \mathrm{~A})$, provoca un efecto negativo para mayores corrientes, donde se genera una mayor oxidación y pérdidas de los elementos de la carga aleante en el revestimiento, haciendo menor su incorporación al depósito metálico.

Se observa, también, que al aumentar la corriente existe una tendencia al aumento en la Tasa de Consumo (Tabla 7). Este comportamiento podría estar asociado a las pérdidas por la descomposición de la caliza que sufre un proceso de disociación endotérmica $\left(\mathrm{CaCO}_{3}=\mathrm{CaO}+\mathrm{CO}_{2}\right)$ en el entorno de $850{ }^{\circ} \mathrm{C}$ y la oxidación de elementos desoxidantes (que se verifica con carácter exotérmico) que también aumenta con la Energía de Entrada provocando un efecto energético compensatorio. Por su parte, la Tasa de Deposición se mantiene prácticamente estable, con una leve tendencia de mayor valor para la corriente de $145 \mathrm{~A}$.

Los resultados de los parámetros de consumo indican que es conveniente trabajar con los menores valores de corriente, ya que se obtiene mayor rendimiento (menores pérdidas) con prácticamente igual tasa de deposición (igual productividad).

La Tabla 8 muestra los valores de incertidumbre obtenidos para la masa consumida, la masa depositada, el rendimiento y las tasas de consumo y de deposición.

Tabla 8. Incertidumbre de las masas consumida y depositada y de los parámetros de consumo $(95 \%)$

\begin{tabular}{c|c|c|c|c|c}
\hline $\mathrm{I}_{\mathrm{s}}(\mathrm{A})$ & & $\mathrm{u}_{\mathrm{c}}$ & $v_{e f}$ & $\mathrm{k}$ & $\mathrm{U}$ \\
\hline \multirow{4}{*}{120} & $\mathrm{~m}_{\mathrm{c}}(\mathrm{g})$ & $0,25 \mathrm{~g}$ & 31,31 & 2,04 & $0,51 \mathrm{~g}$ \\
\cline { 2 - 6 } & $\mathrm{M}_{\mathrm{d}}(\mathrm{g})$ & $0,24 \mathrm{~g}$ & 36,81 & 2,03 & $0,49 \mathrm{~g}$ \\
\cline { 2 - 6 } & $\mathrm{R}(\%)$ & $0,73 \%$ & 1382,24 & 1,96 & $1,43 \%$ \\
\cline { 2 - 6 } & $\mathrm{T}_{\mathrm{c}}(\mathrm{g} / \mathrm{min})$ & $0,17 \mathrm{~g} / \mathrm{min}$ & 100 & 1,98 & $0,34 \mathrm{~g} / \mathrm{min}$ \\
\cline { 2 - 6 } & $\mathrm{T}_{\mathrm{d}}(\mathrm{g} / \mathrm{min})$ & $0,21 \mathrm{~g} / \mathrm{min}$ & 21,85 & 2,84 & $0,60 \mathrm{~g} / \mathrm{min}$ \\
\hline \multirow{5}{*}{145} & $\mathrm{~m}_{\mathrm{c}}(\mathrm{g})$ & $0,26 \mathrm{~g}$ & 28,88 & 2,04 & $0,53 \mathrm{~g}$ \\
\cline { 2 - 6 } & $\mathrm{M}_{\mathrm{d}}(\mathrm{g})$ & $0,26 \mathrm{~g}$ & 28,88 & 2,04 & $0,53 \mathrm{~g}$ \\
\cline { 2 - 6 } & $\mathrm{R}(\%)$ & $0,86 \%$ & 2638,98 & 1,96 & $1,69 \%$ \\
\cline { 2 - 6 } & $\mathrm{T}_{\mathrm{c}}(\mathrm{g} / \mathrm{min})$ & $0,22 \mathrm{~g} / \mathrm{min}$ & 100 & 1,98 & $0,44 \mathrm{~g} / \mathrm{min}$ \\
\cline { 2 - 6 } & $\mathrm{T}_{\mathrm{d}}(\mathrm{g} / \mathrm{min})$ & $0,22 \mathrm{~g} / \mathrm{min}$ & 25,39 & 2,06 & $0,45 \mathrm{~g} / \mathrm{min}$ \\
\hline \multirow{4}{*}{160} & $\mathrm{~m}_{\mathrm{c}}(\mathrm{g})$ & $0,22 \mathrm{~g}$ & 196,00 & 1,96 & $0,44 \mathrm{~g}$ \\
\cline { 2 - 6 } & $\mathrm{M}_{\mathrm{d}}(\mathrm{g})$ & $0,26 \mathrm{~g}$ & 28,88 & 2,04 & $0,53 \mathrm{~g}$ \\
\cline { 2 - 6 } & $\mathrm{R}(\%)$ & $1,21 \%$ & 10250,57 & 1,96 & $2,38 \%$ \\
\cline { 2 - 6 } & $\mathrm{T}_{\mathrm{c}}(\mathrm{g} / \mathrm{min})$ & $0,30 \mathrm{~g} / \mathrm{min}$ & 100 & 1,98 & $0,60 \mathrm{~g} / \mathrm{min}$ \\
\cline { 2 - 6 } & $\mathrm{T}_{\mathrm{d}}(\mathrm{g} / \mathrm{min})$ & $0,35 \mathrm{~g} / \mathrm{min}$ & 161,87 & 1,96 & $0,69 \mathrm{~g} / \mathrm{min}$ \\
\hline
\end{tabular}

A partir de la Tabla 8 se concluye que la incertidumbre expandida asociada a la masa consumida y a la masa depositada varía entre $0,5 \mathrm{~g}$ y $0,6 \mathrm{~g}$, no observándose relación entre los valores de incertidumbre y de corriente. Con relación al rendimiento obsérvese que la incertidumbre expandida varía entre $1,43 \%$ y $2,38 \%$, para la tasa de consumo fueron obtenidos valores entre $0,4 \mathrm{~g} / \mathrm{min}$ y $0,6 \mathrm{~g} / \mathrm{min}$, mientras que para la tasa de deposición la incertidumbre varía de $0,5 \mathrm{~g} / \mathrm{min}$ a $0,7 \mathrm{~g} / \mathrm{min}$.

Los valores de incertidumbre obtenidos pueden ser considerados adecuados para la exactitud de la medición, una vez que representan menos que $4 \%$ del valor medio obtenido. No obstante algunas modificaciones pueden ser introducidas en el proceso de medición de la masa para mejorar la fiabilidad de los parámetros de consumo, entre ellas el aumento del número de lecturas de tres para cinco, obteniéndose así una muestra más representativa.

\section{Conclusiones}

a) El rendimiento muestra un comportamiento decreciente con la corriente, siendo el mejor resultado de $(74,12 \pm 1,43) \%$ para una corriente de soldadura de $120 \mathrm{~A}$. La tasa de consumo crece con el aumento de la corriente presentando $(30,0 \pm$ $0,7) \mathrm{g} / \mathrm{min}$ para $160 \mathrm{~A}$. Por su parte, la tasa de deposición se mantiene prácticamente estable con su mayor valor de $(19,9$ $\pm 0,5) \mathrm{g} / \mathrm{min}$ para la corriente de $145 \mathrm{~A}$.

b) La metodología propuesta permitió evaluar la incertidumbre asociada a los parámetros de consumo de forma simple. Los valores de incertidumbre expandida para el rendimiento varían entre $1,47 \%$ y $2,41 \%$, para la tasa de consumo fueron obtenidos valores entre $0,4 \mathrm{~g} / \mathrm{min}$ y $0,6 \mathrm{~g} / \mathrm{min}$, mientras que para la tasa de deposición la incertidumbre se está entre 0,5 $\mathrm{g} / \mathrm{min}$ y $0,7 \mathrm{~g} / \mathrm{min}$.

c) Las variables que más contribuyeron en la incertidumbre fueron la desviación estándar y la excentricidad de la balanza. Para mejorar la fiabilidad de los parámetros de consumo se recomienda aumentar el tamaño de la muestra.

\section{Agradecimentos}

Los autores agradecen a la FAPEMIG por el apoyo brindado.

\section{Referencias Bibliográficas}

[1] INMETRO, Vocabulário Internacional de Metrologia: Conceitos Fundamentais e Gerais e Termos Associados. 1 ${ }^{\mathrm{a}}$. Edição Luso - Brasileira, Rio de Janeiro, 2012, 95 p.

[2] ASSOCIAÇÃO BRASILEIRA DE MORMAS TÉCNICAS. NBR ISO/IEC 17025, Requisitos Gerais Para Competência de Laboratório de Ensaios e Calibrações. Rio de Janeiro, 2005. 20 p. [3] PARANHOS, R.P.R. A Relevância da Calibração de Sensores na Condução de Experimentos Técnico-Científicos. Soldagem e Inspeção, v.11, n.2, 2006, pp. 117-118.

[4] ISO TAG 4/WG 3, Guide to the Expression of Uncertainty in Measurement, Geneva Switzerland, 2008, 131p.

[5] REIS, R.P. Utilização de Unidades de Medida Segundo o SI em Redações Técnico-Científicas Soldagem e Inspeção, v.11, n.1, 2006, pp.47-52.

[6] INMETRO. Sistema internacional de Unidades - SI, $1^{\text {a }}$ Edição Brasileira da $8^{a}$ Edição do BIPM, Rio de Janeiro. 2012, 94 p. [7] VALDÉS, R.A.; RIBEIRO, J.R.S. Incerteza na Medição da 
Largura de Cordões de Solda. Soldagem e Inspeção, v.14, n.3, 2009, pp. 263-269.

[8] VALDÉS, R.A.; DIAZ CEDRE, E.M.; CRESPO, A.C.; PIRATELLI-FILHO, A. Incerteza na Medição dos Parâmetros Geométricos do Cordão de Solda. Soldagem e Inspeção, v.16, n.1, 2011, pp. 062-070.

[9] VALDÉS, R.A.; CRESPO, A.C.; PIRATELLI-FILHO, A.; DIAZ CEDRE, E.M. Aplicación de Criterios Metrológicos a la Evaluación del Recargue por Soldadura, São Luís, Maranhão, Brasil. CONEM 2012, $10 \mathrm{p}$.

[10] VORNOVITSKY, I.N.; SEMENDYAEV, B.V.; KUCHEROVA, M.I.; ARSYUKOV. Regulating splashing of electrode metal in manual arc welding, Welding International n.21 (2), 2007, pp. 157-159.

[11] FARIAS, J.P.; BÁLSAMO, P.S.; SURIAN, E.S. The effect of wollastonite on operational characteristics of AWS E6013 electrodes. Journal of the Brazilian Society of Mechanical Sciences and Engineering, v.26, n.3, 2004, pp. 17-21.

[12] CRUZ-CRESPO, A.; SCOTTI, A.; RODRIGUEZ-PEREZ, M. Operational behavior assesment of coated tubular electrodes for SMAW hardfacing, Journal of Materials Processing Technology, v.199, 2008, pp. 265-273.

[13] QUINN, T.P.; BRACARENSE, A.Q.; LIU, S. A Meeting rate and temperature distribution model for shielded metal arc welding electrode, Welding Journal n. 12, 1997, pp. 532-538.

[14] BERG, T.; LARIGALDIE, A. Melting rate of covered electrodes, Welding Journal v. 32, n. 5, 1952, pp 268-271.

[15] CRUZ-CRESPO, A.; FUENTES, R.; SCOTTI, A. The Influence of Calcite, Fluorite and Rutile on the Fusion-related Behaviour of Metal Cored Coated Electrodes for Hardfacing. Journal of Materials Engineering and Performance, v. 19 (5), 2010, pp. 685-692.

[16] ORGANIZACIÓN INTERNACIONAL PARA ESTANDARIZACIÓN - ISO 2401-72. Covered Electrodes - Determination of the Efficiency, Metal Recovery and Deposition Coefficient. 1994. 5p.

[17] OGC003, Guia para a Acreditação em Metrologia de Massa. 2005. 10 p.

[18] CENAM. Técnica de trazabilidad e incertidumbre en la magnitud de masa. Calibración de pesas clases, E2, F1, F2, M1, M1-2, M2, M2-3 y M3. México, Revisión 1, Abril de 2008. 17 p. 